\title{
A Comparison of Model Aggregation Methods for Regression
}

\author{
Zafer Barutçuoğlu and Ethem Alpaydın \\ Department of Computer Engineering, Boğaziçi University, Istanbul, Turkey \\ zbarutcu@turk.net, alpaydin@boun.edu.tr
}

\begin{abstract}
Combining machine learning models is a means of improving overall accuracy. Various algorithms have been proposed to create aggregate models from other models, and two popular examples for classification are Bagging and AdaBoost. In this paper we examine their adaptation to regression, and benchmark them on synthetic and real-world data. Our experiments reveal that different types of AdaBoost algorithms require different complexities of base models. They outperform Bagging at their best, but Bagging achieves a consistent level of success with all base models, providing a robust alternative.
\end{abstract}

\section{Introduction}

Combining multiple instances of the same model type is a means for increasing robustness to variance, reducing the overall sensitivity to different starting parameters and noise. Two well-known algorithms for this purpose are Bagging [1] and AdaBoost [23]. Both have been analyzed for classification in much more detail than regression, possibly due to the wider availability of real-life applications. Adapting classification algorithms to regression raises some issues in this setting. In this paper we compare the Bagging algorithm and several AdaBoost variants for regression.

\section{Bagging}

The Bagging (Bootstrap Aggregating) algorithm [1] uses bootstrapping (equiprobable selection with replacement) on the training set to create many varied but overlapping new sets. The base algorithm is used to create a different base model instance for each bootstrap sample, and the ensemble output is the average of all base model outputs for a given input.

The best enhancement by Bagging is when the model instances are very different from each other, since averaging will not have much effect when the outputs are already close. Hence, the most suitable base models for Bagging are unstable models, where small changes in the training set can result in large changes in model parameters. Multilayer perceptrons and regression trees are good candidates.

The particular bootstrap sample size being used has an effect on the performance of Bagging, but the optimal ratio of sample to training set size depends on the data. Instead of manually finetuning this ratio per application, we used validation to automate a coarse adjustment that we named Best-Ratio Bagging. It removes a fraction of the 
training set for validation, performs multiple Bagging instances with different ratios on the remaining training examples, and chooses the Bagging model with the lowest error on the validation set as the final model. Although costly, Best-Ratio Bagging is useful for illustrating the best case performance of Bagging with respect to sample size.

Bagging takes a simple average of outputs, but the evaluation part of AdaBoost can be adopted and a weighted median may be used instead. The weights (confidences) can be calculated as in AdaBoost, using average loss with respect to a loss function. We implemented this variant using linear loss. See Section 3.2 for the computation of confidence and weighted median.

To produce similar but perturbed subsets from one training set, $K$-fold crossvalidation is an alternative to bootstrapping. The training set $\mathcal{X}$ is randomly partitioned into $K$ sets $\mathcal{X}_{i}$ of equal size, and each base model is trained on $\mathcal{X}-\mathcal{X}_{i}$. We called this algorithm Cross-Validation Aggregating (CVA). Evaluation is by averaging outputs, as in Bagging. As opposed to bootstrapping, cross-validation is guaranteed to use all training examples exactly once in exactly $K-1$ subsets. For small $K$, this leads to more efficient use of data than bootstrapping. However as $K$ increases, we get increasingly similar subsets, which should decrease the positive effect of combining.

\section{The AdaBoost Approach}

Since individual bootstrap samples are selected independently, the collective success of the models they produce is through mere redundancy. The boosting approach uses the base models in sequential collaboration, where each new model concentrates more on the examples where the previous models had high error. Different ways of realizing this dynamic focus lead to different algorithms. AdaBoost (Adaptive Boosting) [23] is an efficient and popular implementation of the boosting principle, applied and analyzed with much deeper interest for classification than regression. Since the latter is a more general problem, the basic concept of AdaBoost can be generalized in more than one way for regression.

\subsection{AdaBoost.R}

The originally proposed AdaBoost for regression AdaBoost.R is based on decomposing regression into infinitely many classification tasks [2]. This construction does allow an implementation, but it involves keeping track of a different updatable and integrable loss function for each example. Furthermore, the base learner must be able to accommodate such dynamic loss functions per example. This dynamic-loss approach was also used by Ridgeway et al. [4], but their experiments using naive Bayes base learners yielded no significant justification to afford a per-example redefinable loss, seriously constraining the choice of base learners if not time complexity.

\subsection{Distribution-Based Algorithms}

Drucker's AdaBoost. Drucker's AdaBoost for regression [5] is an ad hoc adaption of the classification AdaBoost. Despite the lack of a rigorous derivation, it uses scalar 
selection probabilities, unlike AdABoost.R. It works much like classification AdaBoost, favoring examples with high error. The ensemble output is the weighted median of the base model outputs, weighted by the models' training confidences.

The weighted median can be computed by first sorting the outputs in order of magnitude, and then summing their weights until the sum exceeds half the weight total. If the weights were integers, this would be analogous to duplicating the outputs by their weights and taking the regular median.

At each step $i$, the algorithm minimizes the error function (in rearranged notation)

$$
J_{i}=\sum_{t=1}^{N} \exp \left(-c_{i}\right) \exp \left(c_{i} L_{i}^{t}\right)
$$

by minimizing per-example losses $L_{i}^{t}$. $c_{i}$ is a measure of confidence over all examples, also used as the combination coefficient during evaluation. Drucker's AdaBoost chooses $c_{i}=\ln \left[\left(1-\bar{L}_{i}\right) / \bar{L}_{i}\right]$ using $\bar{L}_{i}=\sum_{t=1}^{N} L_{i}^{t} p^{t}$ to minimize error, but this appears to be an unjustified adoption of the analytical result for classification. In the experiments we used linear loss (absolute difference) $L=|y-r| / D$ in DRUCKER.AD and square loss $L_{S}=|y-r|^{2} / D^{2}$ in DRUCKER.S where $D=\sup _{t}\left|y^{t}-r^{t}\right|$.

Zemel \& Pitassi's Algorithm. Zemel \& Pitassi [6] provide an algorithm similar to Drucker's, but with alternative mathematical particulars. Here the error function is

$$
J_{i}=\sum_{t=1}^{N} c_{i}^{-1 / 2} \exp \left(c_{i}\left|y_{i}^{t}-r^{t}\right|^{2}\right)
$$

where the loss function is squared error, and not scaled to $[0,1]$.

Although the multiplier is now $c_{i}^{-1 / 2}$, replacing Drucker's $\exp \left(-c_{i}\right)$, with $0<c_{i} \leq 1$ they behave similarly except near zero. Notably Zemel \& Pitassi acknowledge that here $c_{i}$ cannot be analytically determined, and simple line search is used. Finally, this algorithm uses weighted mean instead of weighted median to combine outputs.

We implemented this algorithm as ZeMEL-PitAssi.S and ZeMEL-PITASSI.AD, using the original square loss and linear loss respectively. In ZEMEL-PITASSI.AD we replaced weighted mean by weighted median to match the loss function.

\subsection{Relabeling Algorithms}

Another group of algorithms [78.9], although from different viewpoints, all aim to minimize residual error. In these algorithms each new base model learns artificial labels formed using the per-example training errors (residues) of the current combined model. After training each model $i$ the residues are updated by subtracting the prediction $y_{i}^{t}$ of the new model weighted by its coefficient $c_{i}$. Due to the subtractive training, combination is additive, using a weighted sum. 
The LS_Boost Algorithm. The algorithm LS_Boost is from Friedman's gradient-based boosting strategy [7], using square loss $L=(y-r)^{2} / 2$ where $r$ is the actual training label and $y$ is the current cumulative output $y_{i}=c_{0}+\sum_{j=1}^{i-1} c_{j} h_{j}+c_{i} h_{i}=y_{i-1}+c_{i} h_{i}$. The new training labels $\hat{r}$ should be set to the direction that minimizes the loss, which is the negative gradient with respect to $y$ evaluated at $y_{i-1}$. Then $\hat{r}=[-\partial L / \partial y]_{y=y_{i-1}}=$ $r-y_{i-1}$ which is the current residual error. Substituting into the loss, we get the training error

$$
E=\sum_{t=1}^{N}\left[c_{i} h_{i}^{t}-\hat{r}^{t}\right]
$$

where $\hat{r}^{t}$ are the current residual labels. The combination coefficients $c_{i}$ are determined by solving $\partial E / \partial c_{i}=0$.

Duffy \& Helmbold [8] give an algorithm SQuareLEv.R which is identical in effect. SQuARELEV.C, a variant of SQuARELEv.R, is more interesting in that while also based on residual error, it still uses probabilities. The base learner is fed not the residues $\hat{r}$, but their signs $\operatorname{sign}(\hat{r}) \in\{-1,+1\}$, while the distribution weight of each example is made proportional to $|\hat{r}|$, so each example is still "emphasized" in proportion to its residual error. At the cost of handling probabilities, SQUARELEV.C allows using binary classifiers.

The LAD_Boost Algorithm. The LAD_Boost algorithm from [7] is derived from the same gradient-based framework as LS_BoosT, but using linear loss (absolute deviation). The gradient of linear loss leads to the sign of the residue, so the base models are trained on $\{-1,+1\}$ labels, which also allows using classifiers. Here the derivation of $c_{i}$ yields another weighted median computation. See [7] for details.

\section{Experiment Design}

We tested the algorithms using $J$-leaf regression trees with constant leaf labels. The learner subdivides the leaf having the greatest total squared deviation from the mean, until a specified node count $J$ is reached or all leaves have a single training element. $J$ is used to control base model complexity. Values of $\{2,5,10,15,20\}$ were used for the number of base trees to combine.

Bagging used a fixed 50\% sample size ratio, while Best-Ratio Bagging compared the ratios $10 \%, 20 \%, \ldots, 90 \%$ of the remaining examples for sample size using $50 \%$ of the examples for validation. All experiments were repeated ten times, using $5 \times 2$-fold cross-validation to partition the datasets. The algorithms were compared by the $5 \times 2$-fold cross-validated F test [10] at $95 \%$ confidence. We used the datasets in Table 1 for our experiments. All of them have one-dimensional continuous output for regression.

syndata was synthetically generated for observing the algorithms visually. It has unidimensional input, and on an output range of $[-15,+15]$ it has Gaussian noise of zero mean and unit variance. abalone, boston and calif1000 are from [11]. prostate and birth are from [12]. votes and kin8 datasets are from the StatLib archive of Carnegie Mellon University. For each dataset, we repeated the experiments using $5 \times 2$ fold cross-validation. The error bars in the figures indicate one standard deviation above and below the mean error of the ten runs. 


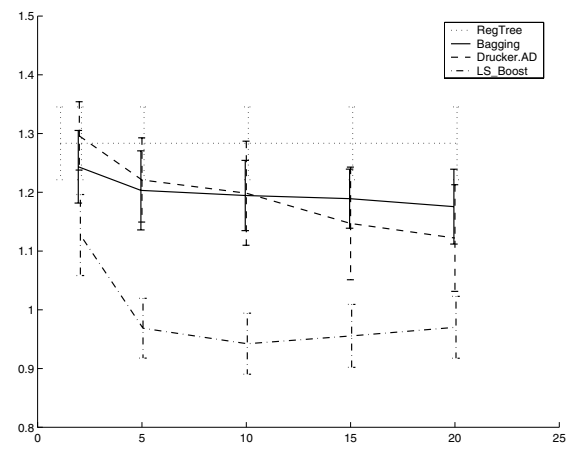

Fig. 1. 5-leaf syndata errors

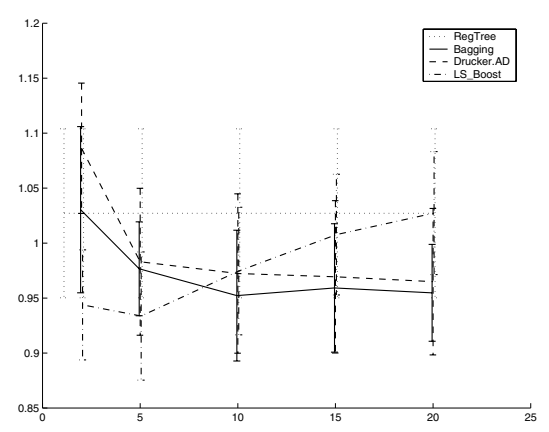

Fig. 2. 10-leaf syndata errors

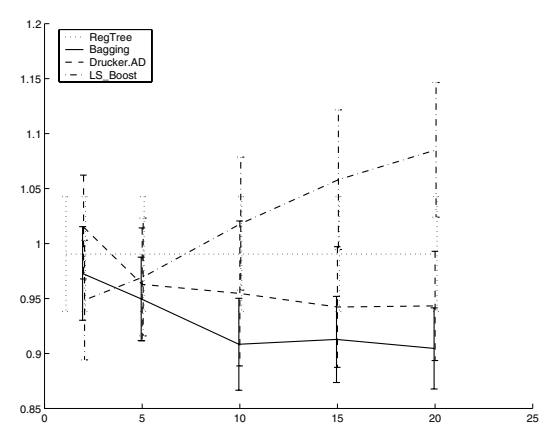

Fig. 3. 15-leaf syndata errors

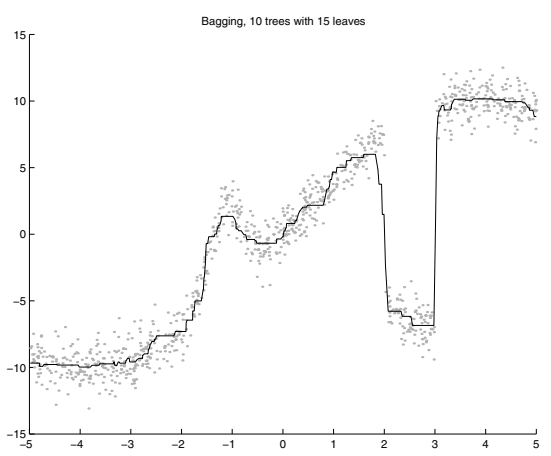

Fig. 4. 15-leaf BAGGING

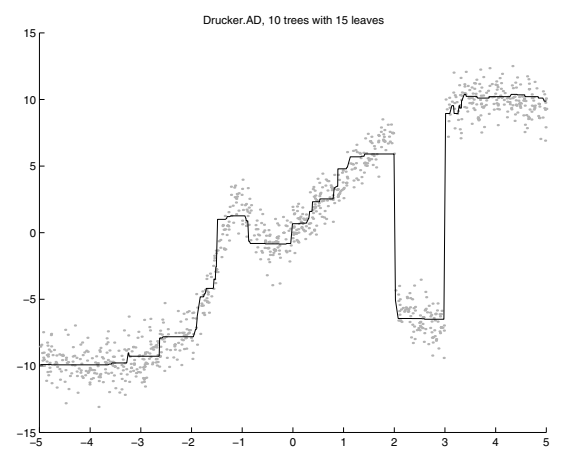

Fig. 5. 15-leaf DrucKer.AD

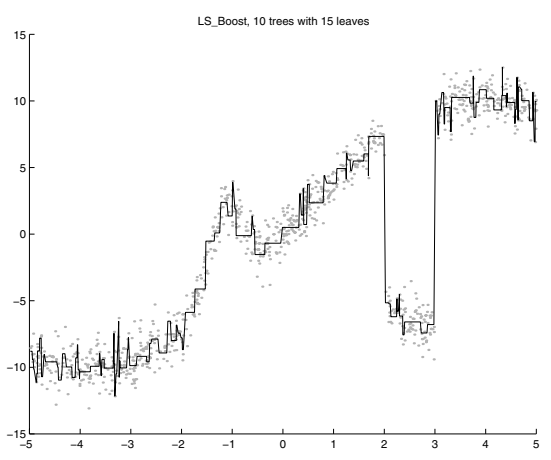

Fig. 6. 15-leaf LS_BoosT 


\section{Simulation Results}

Figures 1 to 3 show the test errors of BAGGING, LS_BoosT and DRUCKER.AD on syndata as the number of trees changes. The unaggregated base algorithm REgTrEe is also included, plotted as constant. These figures illustrate typical behaviors, also observed numerically on other datasets. Figures 45 and 6 show example outputs on syndata using 15-leaf regression trees as base models.

The Bagging methods used both small and large trees with consistent success, although they took a large number of large trees to catch up with the relabeling AdaBoost algorithms. CVA was slightly better than Bagging algorithms for very few base models, and fell behind quickly thereafter as the cross-validated training sets became increasingly similar. W-BAGGING never significantly decreased test error beyond Bagging, sometimes even increasing it. Considering that bootstrap samples are selected uniformly, it is not surprising that "confidence" values derived from accidental differences are bound to disrupt Bagging rather than enhance it. Compared to a fixed 50\% ratio of sample size with BAGGING, BR-BAGGING did not show significant improvement despite the nine-fold execution time.

AdABoost.R, despite its unwieldy time complexity, was not able to improve the base model beyond the statistical significance threshold on any of the datasets.

Drucker's and Zemel \& Pitassi's algorithms did not perform well using small trees on large datasets. They even increased training error, indicating that this is not due to overfitting, but the base models were too coarse to be useful to them.

LAD_BoosT and LS_BoosT started overfitting at much smaller trees than the base algorithm alone, because their modification of labels reduces the complexity of data. This is especially true of LAD_BoosT which greatly simplifies the problem for the base learners by discretizing pseudo-targets to binary. The rapid overfitting can be observed in Figure 6 .

Over the tree sizes used and model counts up to ten, the best instances are reported in Table 2 as average errors and standard deviations over ten runs. The results are compared using the $5 \times 2$-fold cross-validated $F$-test with $95 \%$ confidence on each dataset. Some illustrative pairs of algorithms are shown in Table 3 where the column " $>$ " denotes on how many datasets the left-hand algorithm was significantly superior.

\section{Conclusion}

Bagging proved to be very robust with respect to base model complexity. It was able to reduce test error successfully whether the underlying base models were overfit or underfit. Our variants of Bagging failed to offer any significant improvement over the original BAGGING algorithm, though we did thus verify the integrity of Bagging.

ADABoost.R as it was originally proposed did not show any improvement over the unaggregated base model, let alone BAGGING, despite its special base model requirements for dynamic loss and prohibitive time complexity.

The distribution-based AdaBoost algorithms needed sufficiently complex base models. Otherwise they failed to reduce even the training error. DrUCKER and ZeMEL-PitasSI 
Table 1. Properties of the datasets used

\begin{tabular}{|l|r|r||l|l|r||l|r|r|}
\hline & inputs & size & & inputs & size & & inputs & size \\
\hline syndata & 1 & 1,000 & prostate & 7 & 376 & kin8fh & 8 & 8,192 \\
boston & 12 & 506 & birth & 5 & 488 & kin8nm & 8 & 8,192 \\
calif1000 & 8 & 1,000 & votes & 6 & 3,107 & kin8nh & 8 & 8,192 \\
abalone & 10 & 4,177 & kin8fm & 8 & 8,192 & & & \\
\hline
\end{tabular}

Table 2. Base model and Bagging results

\begin{tabular}{|l|c|c|c|c|c|c|}
\hline & RegTree & Bagging & BR-Bagging & W-Bagging & CVA & AdaBoost.R \\
\hline & avg \pm std & avg \pm std & avg \pm std & avg \pm std & avg \pm std & avg \pm std \\
\hline syndata & $.943 \pm .049$ & $.890 \pm .043$ & $.888 \pm .034$ & $.887 \pm .035$ & $.903 \pm .044$ & $.919 \pm .084$ \\
boston & $.350 \pm .023$ & $.295 \pm .016$ & $.294 \pm .026$ & $.296 \pm .019$ & $.308 \pm .028$ & $.307 \pm .028$ \\
calif1000 & $.506 \pm .028$ & $.445 \pm .016$ & $.444 \pm .017$ & $.436 \pm .019$ & $.452 \pm .018$ & $.464 \pm .022$ \\
votes & $.493 \pm .013$ & $.444 \pm .006$ & $.445 \pm .005$ & $.446 \pm .004$ & $.458 \pm .007$ & \\
prostate & $.668 \pm .072$ & $.635 \pm .042$ & $.642 \pm .035$ & $.605 \pm .044$ & $.627 \pm .053$ & \\
birth & $.812 \pm .055$ & $.780 \pm .033$ & $.777 \pm .029$ & $.781 \pm .031$ & $.785 \pm .030$ & \\
abalone & $.545 \pm .005$ & $.521 \pm .018$ & $.513 \pm .011$ & $.483 \pm .008$ & $.523 \pm .015$ & \\
kin8fm & $.439 \pm .003$ & $.316 \pm .006$ & $.314 \pm .008$ & $.330 \pm .007$ & $.358 \pm .009$ & \\
kin8fh & $.553 \pm .005$ & $.457 \pm .005$ & $.456 \pm .007$ & $.464 \pm .005$ & $.488 \pm .008$ & \\
kin8nm & $.595 \pm .012$ & $.523 \pm .004$ & $.523 \pm .003$ & $.511 \pm .007$ & $.546 \pm .006$ & \\
kin8nh & $.657 \pm .011$ & $.601 \pm .007$ & $.600 \pm .008$ & $.597 \pm .007$ & $.615 \pm .009$ & \\
\hline & Drucker.AD & Drucker.S & Z\&P.AD & Z\&P.S & LAD_Boost & LS_Boost \\
\hline & avg \pm std & avg \pm std & avg. \pm std & avg \pm std & avg \pm std & avg \pm std \\
\hline syndata & $.917 \pm .055$ & $.921 \pm .061$ & $.910 \pm .039$ & $.900 \pm .045$ & $.978 \pm .050$ & $.934 \pm .058$ \\
boston & $.276 \pm .019$ & $.297 \pm .014$ & $.280 \pm .018$ & $.286 \pm .023$ & $.346 \pm .025$ & $.335 \pm .029$ \\
calif1000 & $.429 \pm .015$ & $.457 \pm .016$ & $.425 \pm .020$ & $.447 \pm .019$ & $.455 \pm .021$ & $.468 \pm .016$ \\
votes & $.443 \pm .006$ & $.455 \pm .005$ & $.447 \pm .004$ & $.449 \pm .006$ & $.472 \pm .012$ & $.481 \pm .014$ \\
prostate & $.650 \pm .051$ & $.678 \pm .039$ & $.650 \pm .075$ & $.631 \pm .049$ & $.600 \pm .023$ & $.678 \pm .053$ \\
birth & $.791 \pm .026$ & $.792 \pm .034$ & $.790 \pm .026$ & $.790 \pm .026$ & $.793 \pm .028$ & $.783 \pm .028$ \\
abalone & $.514 \pm .015$ & $.544 \pm .029$ & $.497 \pm .011$ & $.544 \pm .039$ & $.497 \pm .005$ & $.520 \pm .008$ \\
kin8fm & $.288 \pm .005$ & $.279 \pm .006$ & $.294 \pm .005$ & $.295 \pm .007$ & $.316 \pm .012$ & $.296 \pm .011$ \\
kin8fh & $.444 \pm .006$ & $.438 \pm .004$ & $.446 \pm .004$ & $.446 \pm .005$ & $.481 \pm .005$ & $.485 \pm .004$ \\
kin8nm & $.502 \pm .004$ & $.523 \pm .007$ & $.510 \pm .005$ & $.523 \pm .004$ & $.531 \pm .009$ & $.528 \pm .012$ \\
kin8nh & $.597 \pm .011$ & $.600 \pm .012 .601 \pm .009$ & $.604 \pm .012$ & $.627 \pm .011$ & $.624 \pm .010$ \\
\hline
\end{tabular}

Table 3. Significant superiority over 11 datasets

\begin{tabular}{|c|c|c|c|c|c|c|c|c|c|}
\hline & & $>$ & $=$ & $<$ & & & $>$ & $=$ & $<$ \\
\hline DRUCKER.AD & ZP.AD & 1 & 10 & 0 & DRUCKER.S & REGTREE & 5 & 6 & 0 \\
\hline DRUCKER.S & ZP.S & 2 & 8 & 1 & W-BAGGING & BAGGING & 0 & 9 & \\
\hline DRUCKER.AD & LAD_BoosT & 4 & 7 & 0 & BR-BAGGING & BAGGING & 0 & 11 & \\
\hline DRUCKER.S & LS_BoosT & 2 & 9 & 0 & LAD_BoosT & REGTREE & 6 & 5 & \\
\hline BAGGING & RegTree & 7 & 4 & 0 & LS_BoosT & RegTree & 5 & 6 & \\
\hline BAGGING & CVA & 3 & 8 & 0 & LAD_Boost & BAGGING & 0 & 9 & \\
\hline DRUCKER.AD & REGTreE & 7 & 4 & 0 & LS_BoosT & BAGGING & 0 & 7 & \\
\hline
\end{tabular}


were almost always equal in performance. In that case DRUCKER may be slightly more preferable, considering the inconvenient line search in ZEMEL-PITASSI.

The relabeling AdaBoost algorithms, in contrast, called for very simple models that would normally underfit. With complex base models their performance deteriorated rapidly as they started overfitting the data.

In selecting an aggregation algorithm for a regression task, if the base models are inherently simple or their complexity can be adjusted by some validation method, the relabeling algorithms should be considered, since they can provide the best accuracy using the fewest base models. If the models cannot be prevented from overfitting, one of the distribution-based AdaBoost algorithms can be used. The choice of loss function depends on the data at hand. If one algorithm must be selected to handle both simple and complex base models, Bagging is a safe bet.

Acknowledgments. This work has been supported by Boğaziçi University Scientific Research Project 02A104D and the Turkish Academy of Sciences, in the framework of the Young Scientist Award Program (EA-TÜBA-GEBIP/2001-1-1).

\section{References}

1. Breiman, L., "Bagging Predictors", Machine Learning, Vol. 24, No. 2, pp. 123-140, 1996.

2. Freund, Y. and R. E. Schapire, "A Decision-Theoretic Generalization of On-line Learning and an Application to Boosting", European Conf. on Computational Learning Theory, pp. 23-37, 1995.

3. Freund, Y. and R. E. Schapire, "Experiments with a New Boosting Algorithm", International Conf. on Machine Learning, pp. 148-156, 1996.

4. Ridgeway, G., D. Madigan and T. Richardson, "Boosting methodology for regression problems", Proc. of Artificial Intelligence and Statistics, pp. 152-161, 1999.

5. Drucker, H., "Improving regressors using boosting techniques", Proc. 14th International Conf. on Machine Learning, pp. 107-115, Morgan Kaufmann, San Francisco, CA, 1997.

6. Zemel, R. S. and T. Pitassi, "A Gradient-Based Boosting Algorithm for Regression Problems", Adv. in Neural Information Processing Systems, Vol. 13, 2001.

7. Friedman, J. H., Greedy Function Approximation: a Gradient Boosting Machine, Tech. Rep. 7, Stanford University, Dept. of Statistics, 1999.

8. Duffy, N. and D. Helmbold, "Leveraging for Regression", Proc. 13th Annual Conf. on Computational Learning Theory, pp. 208-219, Morgan Kaufmann, San Francisco, CA, 2000.

9. Rätsch, G., M. Warmuth, S. Mika, T. Onoda, S. Lemm and K.-R. Müller, "Barrier Boosting", Proc. 13th Annual Conference on Computational Learning Theory, 2000.

10. Alpaydın, E., "Combined $5 \times 2 \mathrm{cv} F$ Test for Comparing Supervised Classification Learning Algorithms", Neural Computation, Vol. 11, No. 8, pp. 1885-1992, 1999.

11. Blake, C. and P. M. Murphy, "UCI Repository of Machine Learning Databases", http://www.ics.uci.edu/〜mlearn/MLRepository.html

12. Hosmer, D. and S. Lemeshow, Applied Logistic Regression, John Wiley \& Sons Inc., 2nd edn., 2000. 